\title{
The masses of heavy pentaquarks in the non-relativistic Bethe-Salpeter quark model
}

\author{
M. Abu-Shady ${ }^{a}$, N. H. Gerish ${ }^{b}$ and M. M. A. Ahmed ${ }^{c}$ \\ ${ }^{a}$ Department of Mathematics and Computer Science, Faculty of Science, Menoufia University, Egypt. \\ ${ }^{b}$ Department of Mathematics and Computer Science, Faculty of Science, Suez Canal University, Egypt. \\ ${ }^{c}$ Department of Mathematics and Computer Science, Faculty of Science, Al-Azhar University, Egypt.
}

Received 1 May 2021; accepted 5 July 2021

\begin{abstract}
The exotic particles such as the pentaquarks are believed to strengthen the understanding of important interactions and the principle of QCD in which pentaquarks contain two heavy-valence quarks. The structure of two bodies including an antiquark and two-diquark is introduced. A new potential for quark interaction is suggested which includes the logarithm and linear potentials, as well as the spin-spin interaction. The suggested potential is included in the framework of spinless of the Bethe-Salpeter equation. A comparison with other works is presented which provides a good description of pentaquarks.
\end{abstract}

Keywords: Pentaquarks; antiquark; two-diquark.

DOI: https://doi.org/10.31349/RevMexFis.68.010801

\section{Introduction}

Mesons and baryons (and so multiquark states) can be described by QCD models, in a medium [1-8]. Diquark interactions appear to play an important role in hadron physics, according to QCD [9], the only requirement for these states is to be color singlets. The quark model was suggested by Murray Gell-Mann and George Zweig in their papers in 1964 since exotic hadrons such as pentaquarks were discussed [10]. While most ground-state mesons and baryons are experimentally well defined, several recently observed states are under debate because their quark content and/or spin/parity are unknown; see [11-15] for a study on possible exotic states. Previously, exotic particles like X(3872) and $Z_{c}$ have been investigated by scientists [16]. The decay of $Z_{c}$ into $J / \psi$ (a bound state of charm-anticharm quarks) revealed that this particle was made up of two quarks and two antiquarks. The tetraquark as a bound state is supporting the existence of five-quark states called pentaquarks $[17,18]$.

The authors $[19,20]$ study the spectra of tetraquarks and present the fully charmed tetraquark decay width. The physics of pentaquarks has recently improved in this regard, with the $\mathrm{LHCb}$ Collaboration reporting three heavy pentaquarks, $P_{c}$ 's. An experimental observation of heavy pentaquarks containing charm or bottom quarks has also been looked into [21].

The binding mechanism associated with exotic states is still unclear. Various interpretations can be assigned according to the following three types of models. (i) The mesonbaryon molecular model or their mixture. For this model, the energy spectrum has been evaluated using a chiral effective Lagrangian approach, the QCD sum rules, the color-screen model, and the scattering amplitudes approach. (ii) Diquark (triquark) interaction models, for which the diquark-diquarkantiquark model and compact diquark-triquark model have been used; see [22] and references therein. In addition, a classification of all possible pentaquark states QQqq $\bar{q}$ depend on the corresponding quantum numbers and mass predictions have been presented [23, 24].

The LHCb collaboration reported [25] the observation of a narrow pentaquark state, $P_{c}(4312)^{+}$, a decay of $J / \psi$ with the statistical significance of $7.3 \sigma$ in a data sample of $\Lambda_{b}^{0} \rightarrow J / \psi+p+K^{-}$decays, one order of magnitude higher than the previous collaboration study at the $\mathrm{LHCb}$ [26]. The pentaquark structure of $P_{c}(4450)^{+}$, previously presented by $\mathrm{LHCb}$, is also observed and reported [25,27] to consist of two main small overlapping peaks, $P_{c}(4440)^{+}$and $P_{c}(4457)^{+}$, where this two-peak representation has a statistical significance of $5.4 \sigma$. Until now, for the features of pentaquarks, many various schemes have been suggested. For example [28], two interpolating currents are used in a diquarkdiquark-antiquark description of pentaquarks to measure the mass of the $E^{--}$state in the form of the QCD sum rules. With the perturbative chiral quark model [29], the mass spectra of $J^{p}=3 / 2$ pentaquarks were examined. In Ref. [30], the authors calculated pentaquark masses by a changed mass formula adapted to the masses of baryons. In a constituent quark model with a full description of qqqq $\bar{q}$ pentaquark states, some properties of qqqq $\bar{q}$ pentaquark states, like their masses and magnetic moments, were acquired [31]. Karliner and Lipkin [32]. proposed a formula to estimate their masses for pentaquark states. The diquark-triquark model was applied to clarify charmonium-pentaquark states [22]. The important characteristics are based on the antiquark-diquarkdiquark scheme, that correlates to the experimental evidence obtained [33]. The QQqq $\bar{q}$ pentaquarks were studied in a potential model, based on the hypothesis that they are composite particles containing two diquarks and one antiquark with a Cornell potential as $V_{\text {Cornell }}=(-a / r)+b r+c$ [34]. The same potential was used in Ref. [35] but the authors developed the 
pentaquark model using multiple hypotheses.

In this study, we determine the masses of pentaquarks in the ground state, considering that a pentaquark is a bound state of two diquarks and an antiquark. The physical theory behind the description of the diquark is the union to combine any two quarks into a colored quasi-bound state. This approach makes it possible, firstly, to discuss the idea of using the diquark principle in this case. Secondly, to calculate more brief details for the masses, expressions. We used the Bethe-Salpeter equation with the potential energy of quark interaction given by logarithm, lienar, and spindependent potentials. To the best of our knowledge, the logarithm potential has not been used to calculate the masses of pentaquarks. Our numerical results for the ground masses for the various pentaquarks models with spin $(1 / 2)^{-},(3 / 2)^{-}$and $(5 / 2)^{-}$are provided. Our observations can be correlated with the outcomes of other studies, such as [34,35].

The paper is arranged as follows: in Sec. 2, the BetheSalpeter equation is resolved in the present potential. In Sec. 3, the numerical results and discussion are shown. In Sec. 4 , the conclusion is presented.

\section{The Theoretical Model}

To research two heavy pentaquarks, we assume them to be the bound state of two heavy diquarks and antiquarks. As a result, the Bethe-Salpeter equation in QCD can be used to describe this two-body structure. The well-known BethSalpeter equation is obtained by considering the natural units (where $\bar{h}=c=1$ ) [35],

$$
\begin{aligned}
& {\left[\left(p^{2}+m_{1}^{2}\right)^{1 / 2}+\left(p^{2}+m_{2}^{2}\right)^{1 / 2}\right.} \\
& +V(r)] \psi_{n l}(r)=M \psi_{n l}(r),
\end{aligned}
$$

where $m_{1}$ and $m_{2}$ describe the masses of the two-body structure components, $V(r)$ is the quark-antiquark potential, $M$ is the mass of the bound state and $\psi$ denotes the wave function. A convenient solution to avoid interacting with the kinetic energy operator's non-local character in Eq. (1) is to extend it, as in [36] for heavy interacting particles at $p<\mu$,

$$
\begin{gathered}
\left(p^{2}+m_{1}^{2}\right)^{1 / 2}+\left(p^{2}+m_{2}^{2}\right)^{1 / 2}=m_{1}+m_{2} \\
+\frac{p^{2}}{2 \mu}-\frac{p^{2}}{8 \alpha \mu^{3}}+\ldots
\end{gathered}
$$

where the reduced mass $\mu$ is

$$
\mu=\frac{m_{1} m_{2}}{m_{1}+m_{2}}
$$

and

$$
\alpha=\frac{m_{1} m_{2}}{m_{1} m_{2}-3 \mu^{2}} .
$$

We only take terms up to order $1 / \mu^{3}$ in the above equation; this is a strong approximation for heavy interacting particles and uses the operator $p^{2}=-\nabla^{2}$ and $p^{4}=4 \mu^{2}[E-V(r)]^{2}$, where the energy of the bound state is $E=M-m_{1}-m_{2}$, it with which it is possible to rewrite Eq. (1) in

$$
\begin{gathered}
\left\{-\frac{1}{2 \mu}\left[\frac{d^{2}}{d r^{2}}+\frac{2}{r} \frac{d}{d r}-\frac{l(l-1)}{r^{2}}\right]+V(r)+m_{1}+m_{2}\right. \\
\left.-\frac{\left[M-m_{1}-m_{2}-V(r)\right]^{2}}{2 \alpha \mu}\right\} \psi_{n l}(r)=M \psi_{n l}(r) .
\end{gathered}
$$

The potential used in Eq. (3) for each two-body interaction contains the logarithm and linear potentials with a spinspin interaction as

$$
\begin{aligned}
V(r) & =V_{\log }(r)+V_{\text {spin }}(r), \\
V_{\log }(r) & =a r+b \log \frac{r}{r_{0}}, \\
V_{\text {spin }}(r) & =\eta e^{-\sigma^{2} r^{2}}, \quad \eta=\frac{A \sigma^{3} S_{1} \cdot S_{2}}{2 \pi^{3 / 2} m_{1} m_{2}}
\end{aligned}
$$

The parameter here specifies the function of the smeared delta for that we follow $\sigma=1.209 \mathrm{GeV}$ [34] and $r_{0}=7.959 \mathrm{GeV}^{-1}$ [37]. In the one gluon exchange approximation, the parameter $A$ is related to the strong constant coupling $\alpha_{s}$.

$S_{1}$ and $S_{2}$ are the spins of the interacting particles in the spin-spin interaction, where: $S_{1} \cdot S_{2}=(1 / 2)[S(S+1)-$ $\left.S_{1}\left(S_{1}+1\right)-S_{2}\left(S_{2}+1\right)\right]$, and $S$ is the total spin.

Two contributions are usually included in the interaction potential of two colored objects. One of them is based on the $q \bar{q}$ potential determined in the gauge/string duality technique in QCD, and another concept defines the interaction between spin-spin. Color interaction describes this potential that is determined by a virtual model inspired by Cornell potential presented in Ref. [34] and that it has been replaced the logarithmic potential in present work as in Eq. (5). A comparison between both potentials is shown in Fig. 1, where it is clear that one has the same behavor for the two potentials.

By introducing $\psi_{n l}(r)=X(r) r^{-1}$ and using the potential in Eq. (4), then Eq. (3) can defined as;

$$
\begin{aligned}
-\frac{d^{2} X}{d r^{2}} & +\left(\frac{l[l-1]}{r^{2}}-\frac{V^{2}(r)}{\alpha}\right. \\
& +V(r)\left[\frac{2\left(M-m_{1}-m_{2}\right)}{\alpha}+2 \mu\right]-\frac{\left[M-m_{1}-m_{2}\right]^{2}}{\alpha} \\
& \left.+2 \mu\left(-M+m_{1}+m_{2}\right)\right\} X(r)=0
\end{aligned}
$$

Taking into account that $r<1 \mathrm{fm}$, we apply the simple approximation to analytically solve the above equation. Approximating $e^{-\sigma^{2} r^{2}} \approx 1-\sigma^{2} r^{2}+\ldots$ and $\log \left[r / r_{0}\right] \approx$ $-(3 / 2)+\left(2 / r_{0}\right) r-\left(1 / 2 r_{0}^{2}\right) r^{2}+\ldots$ leading to a potential parametrized by

$$
V(r)=a_{1}+a_{2} r+a_{3} r^{2}
$$




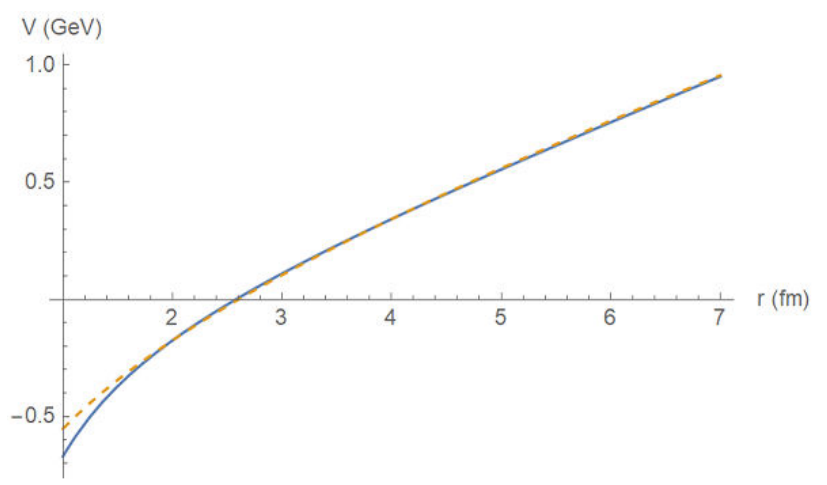

FIGURE 1. A comparison between the Cornell [35] and logarithm potentials.

where

$$
\begin{aligned}
& a_{1}=-\frac{3 b}{2}+\eta, \\
& a_{2}=a+\frac{2 b}{r_{0}}, \\
& a_{3}=-\frac{b}{2 r_{0}^{2}}-\eta \sigma^{2},
\end{aligned}
$$

with which Eq. (7) becomes

$$
\begin{aligned}
\frac{d^{2} X(r)}{d r^{2}} & \approx\left(\zeta_{1} r^{4}+\zeta_{2} r^{3}\right. \\
& \left.+\zeta_{3} r^{2}+\zeta_{4} r+\zeta_{5}+\frac{\zeta_{6}}{r^{2}}\right) X(r),
\end{aligned}
$$

where

$$
\begin{aligned}
& \zeta_{1}=-\frac{a_{3}^{2}}{\alpha} \\
& \zeta_{2}=-\frac{2 a_{2} a_{3}}{\alpha} \\
& \zeta_{3}=-\frac{a_{2}^{2}+2 a_{1} a_{3}}{\alpha}+a_{3}\left(\frac{2\left[M-m_{1}-m_{2}\right]}{\alpha}+2 \mu\right) \\
& \zeta_{4}=-\frac{2 a_{1} a_{2}}{\alpha}+a_{2}\left(\frac{2\left[M-m_{1}-m_{2}\right]}{\alpha}+2 \mu\right) \\
& \zeta_{5}=-\frac{a_{1}^{2}}{\alpha}+a_{1}\left(\frac{2\left[M-m_{1}-m_{2}\right]}{\alpha}+2 \mu\right) \\
& \zeta_{6}=l(l-1) .
\end{aligned}
$$

Equation (10) cannot be solved analytically yet, so the following method suggested [38] is used in which we describe

$$
X_{n l}(r)=\Omega(r) e^{\phi(r)},
$$

where

$$
\begin{aligned}
\phi(r) & =t_{0}+t_{1} r^{2}+t_{2} r^{3}+t_{3} \ln [r] \\
X^{\prime}(r) & =\left(9 t_{2}^{2} r^{4}+12 t_{1} t_{2} r^{3}+4 t_{1}^{2} r^{2}+\left[6 t_{2} t_{3}+6 t_{2}\right] r\right. \\
& \left.+\left[4 t_{1} t_{3}+2 t_{1}\right]+\left[t_{3}^{2}-t_{3}\right] \frac{1}{r^{2}}\right) X(r)
\end{aligned}
$$
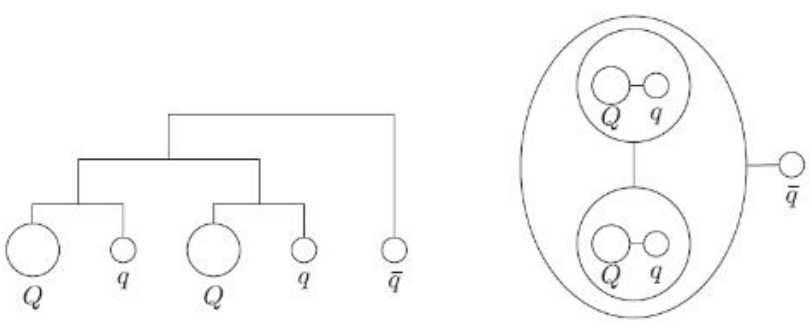

FIGURE 2. The form of pentaquark QqQq $\bar{q}$.

with

$$
\begin{aligned}
t_{1} & = \pm \frac{\sqrt{\zeta_{3}}}{2} \\
t_{2} & = \pm \frac{\sqrt{\zeta_{1}}}{3} \\
t_{3} & =\frac{\zeta_{4}}{2 \sqrt{\zeta_{1}}}-1,
\end{aligned}
$$

we then get

$$
\zeta_{5}= \pm \sqrt{\zeta_{3}}\left\{\frac{ \pm \zeta_{4}}{\sqrt{\zeta_{1}}}-1\right\}
$$

We solve Eqs. (15)-(17) with Eq. (11), where we have used the masses of quark as $m_{u}=m_{d}=0.302 \mathrm{GeV}$, $m_{c}=1.733 \mathrm{GeV}, m_{s}=0.454 \mathrm{GeV}, m_{b}=5.139 \mathrm{GeV}$, $A_{c}=7.920$ and $A_{b}=3.087$ [35]. We calculate the diquark masses as shown in Table I, the following notation is assumed to be: $[Q q]$ diquark has spin 0 , and $\{Q q\}$ for spin 1 .

\section{Numerical results and discussion}

It's worth noting that the one-gluon-exchange approximation and the use of an instantaneous potential can only be extended to heavy states in which at least one of the two interacting particles is heavy, containing a charm or bottom quark. As a result, we calculate the masses of pentaquarks with at least one heavy quark. Furthermore, we only consider states with orbital angular momentum at each state $l=0$.

TABLE I. The diquark masses (in $\mathrm{GeV}$ ).

\begin{tabular}{lccc}
\hline Diquark & $M$ (this work) & $M[34]$ & $M[35]$ \\
\hline$[\mathrm{bu}]_{s=0}$ & 5.24 & 5.513 & 5.29 \\
$\{\mathrm{bu}\}_{s=1}$ & 5.55 & 5.53 & 5.438 \\
{$[\mathrm{bs}]_{s=0}$} & 5.56 & 5.62 & 5.411 \\
$\{\mathrm{bs}\}_{s=1}$ & 5.59 & 5.63 & 5.572 \\
{$[\mathrm{cu}]_{s=0}$} & 2.16 & 2.118 & 1.904 \\
$\{\mathrm{cu}\}_{s=1}$ & 2.45 & 2.168 & 2.322 \\
{$[\mathrm{cs}]_{s=0}$} & 2.56 & 2.23 & 1.966 \\
$\{\mathrm{cs}\}_{s=1}$ & 2.6 & 2.37 & 2.442 \\
\hline
\end{tabular}


TABLE II. The masses of pentaquarks at $J^{p}=(1 / 2)^{-}$in $\mathrm{GeV}$.

\begin{tabular}{cccc}
\hline Pentaqurk & $M$ (this work) & $M[34]$ & $M[35]$ \\
\hline$[\mathrm{bu}]_{s=1}[\mathrm{bu}]_{s=0} \bar{u}$ & 11.12 & 11.19 & 11.17 \\
{$[\mathrm{bs}]_{s=1}[\mathrm{bu}]_{s=0} \bar{u}$} & 11.15 & 11.28 & 11.16 \\
{$[\mathrm{bs}]_{s=0}[\mathrm{bu}]_{s=1} \bar{u}$} & 11.44 & 11.28 & 11.29 \\
{$[\mathrm{cu}]_{s=0}[\mathrm{cu}]_{s=1} \bar{u}$} & 4.21 & 4.64 & 4.33 \\
{$[\mathrm{cs}]_{s=1}[\mathrm{cu}]_{s=0} \bar{u}$} & 4.38 & 4.73 & 4.4 \\
{$[\mathrm{cs}]_{s=0}[\mathrm{cu}]_{s=1} \bar{u}$} & 4.67 & 4.73 & 4.64 \\
{$[\mathrm{bu}]_{s=0}[\mathrm{cu}]_{s=1} \bar{u}$} & 7.9 & 7.94 & 7.67 \\
{$[\mathrm{bu}]_{s=1}[\mathrm{cu}]_{s=0} \bar{u}$} & 7.91 & 7.94 & 7.60 \\
{$[\mathrm{bu}]_{s=0}[\mathrm{cs}]_{s=1} \bar{u}$} & 8.06 & 8.04 & 7.80 \\
{$[\mathrm{bu}]_{s=1}[\mathrm{cs}]_{s=0} \bar{u}$} & 8.33 & 8.04 & 7.76 \\
\hline
\end{tabular}

TABLE III. The masses of pentaquarks at $J^{p}=(3 / 2)^{-}$in GeV.

\begin{tabular}{cccc}
\hline Pentaqurk & $M$ (this work) & $M[34]$ & $M[35]$ \\
\hline$[\mathrm{bu}]_{s=1}[\mathrm{bu}]_{s=1} \bar{u}$ & 11.24 & 11.18 & 11.47 \\
{$[\mathrm{bs}]_{s=1}[\mathrm{bu}]_{s=1} \bar{u}$} & 11.28 & 11.27 & 11.60 \\
{$[\mathrm{bu}]_{s=0}[\mathrm{bu}]_{s=1} \bar{u}$} & 11.47 & 11.21 & 11.32 \\
{$[\mathrm{bs}]_{s=1}[\mathrm{bu}]_{s=0} \bar{u}$} & 11.49 & 11.30 & 11.45 \\
{$[\mathrm{bs}]_{s=0}[\mathrm{bu}]_{s=1} \bar{u}$} & 11.78 & 11.30 & 11.44 \\
{$[\mathrm{cs}]_{s=1}[\mathrm{cu}]_{s=1} \bar{u}$} & 5.35 & 4.72 & 4.81 \\
{$[\mathrm{cu}]_{s=1}[\mathrm{cu}]_{s=1} \bar{u}$} & 5.45 & 4.62 & 4.76 \\
{$[\mathrm{cu}]_{s=0}[\mathrm{cu}]_{s=1} \bar{u}$} & 5.58 & 4.72 & 4.87 \\
{$[\mathrm{cs}]_{s=1}[\mathrm{cu}]_{s=0} \bar{u}$} & 5.72 & 4.82 & 4.98 \\
{$[\mathrm{cs}]_{s=0}[\mathrm{cu}]_{s=1} \bar{u}$} & 5.95 & 4.82 & 4.87 \\
{$[\mathrm{bu}]_{s=0}[\mathrm{cu}]_{s=1} \bar{u}$} & 8.29 & 7.97 & 8.23 \\
{$[\mathrm{bu}]_{s=1}[\mathrm{cu}]_{s=0} \bar{u}$} & 8.299 & 7.97 & 7.76 \\
{$[\mathrm{bu}]_{s=1}[\mathrm{cu}]_{s=1} \bar{u}$} & 8.45 & 7.97 & 8.37 \\
{$[\mathrm{bu}]_{s=0}[\mathrm{cs}]_{s=1} \bar{u}$} & 8.46 & 8.06 & 8.34 \\
{$[\mathrm{bu}]_{s=1}[\mathrm{cs}]_{s=0} \bar{u}$} & 8.56 & 8.06 & 7.92 \\
{$[\mathrm{bu}]_{s=1}[\mathrm{cs}]_{s=1} \bar{u}$} & 8.63 & 8.03 & 8.49 \\
\hline
\end{tabular}

TABLE IV. The masses of pentaquarks at $J^{p}=(5 / 2)^{-}$in GeV.

\begin{tabular}{cccc}
\hline Pentaqurk & $M$ (this work) & $M[34]$ & $M[35]$ \\
\hline$[\mathrm{bu}]_{s=1}[\mathrm{bu}]_{s=1} \bar{u}$ & 11.18 & 11.22 & 11.69 \\
{$[\mathrm{bs}]_{s=1}[\mathrm{bu}]_{s=1} \bar{u}$} & 11.21 & 11.31 & 11.74 \\
{$[\mathrm{cs}]_{s=1}[\mathrm{cu}]_{s=1} \bar{u}$} & 4.77 & 4.85 & 4.98 \\
{$[\mathrm{cu}]_{s=1}[\mathrm{cu}]_{s=1} \bar{u}$} & 4.85 & 4.75 & 4.87 \\
{$[\mathrm{bu}]_{s=1}[\mathrm{cu}]_{s=1} \bar{u}$} & 8.73 & 7.98 & 8.1 \\
{$[\mathrm{bu}]_{s=1}[\mathrm{cs}]_{s=1} \bar{u}$} & 8.94 & 8.07 & 8.64 \\
\hline
\end{tabular}

The masses of pentaquarks in the ground state are calculated using the hypothesis that a pentaquark is a bound state of two diquarks and an antiquark. The union of any two quarks to form a colored quasi-bound state is the physical theory behind the diquark's explanation. This method allows us to first explore the concept of applying the diquark theory in this situation. Second, to obtain more concise information for the masses expressions. By including the logarithm, linear and the spin-dependent potentials, we solve the Bethe-Salpeter equation of quark interaction. Using the relationship between logarithmic potential and the Cornell potential [35] $V_{\text {Cornell }}=(-a / r)+b r+c$, we get $a=0.14 \mathrm{GeV}$ and $b=0.342 \mathrm{GeV}^{2}$ as the parameters of present potential. We present our numerical results for ground state masses for the pentaquarks model stated in Fig. 2 and with spin $(1 / 2)^{-}$, $(3 / 2)^{-}$and $(5 / 2)^{-}$.

The masses of pentaquarks with two heavy diquarks are determined, with each diquark having one light quark and one heavy quark, as shown in Fig. 2. We measure heavy diquark

TABLE V. The masses of pentaquarks at $J^{p}=(1 / 2)^{-}$considering $\bar{q}=\bar{c}$ or $\bar{b}$ in $\mathrm{GeV}$.

\begin{tabular}{cccc}
\hline Pentaquark & $\begin{array}{c}M \\
\text { (this work) }\end{array}$ & Pentaquark & $\begin{array}{c}M \\
\text { (this work) }\end{array}$ \\
\hline$[\mathrm{cu}]_{s=1}[\mathrm{cu}]_{s=0} \bar{c}$ & 7.17 & {$[\mathrm{cu}]_{s=1}[\mathrm{cu}]_{s=0} \bar{b}$} & 10.65 \\
{$[\mathrm{cu}]_{s=0}[\mathrm{cs}]_{s=1} \bar{c}$} & 7.31 & {$[\mathrm{cu}]_{s=0}[\mathrm{cs}]_{s=1} \bar{b}$} & 10.803 \\
{$[\mathrm{cu}]_{s=1}[\mathrm{cs}]_{s=0} \bar{c}$} & 7.56 & {$[\mathrm{cu}]_{s=1}[\mathrm{cs}]_{s=0} \bar{b}$} & 11.06 \\
{$[\mathrm{cs}]_{s=1}[\mathrm{cs}]_{s=0} \bar{c}$} & 7.72 & {$[\mathrm{cs}]_{s=1}[\mathrm{cs}]_{s=0} \bar{b}$} & 11.21 \\
{$[\mathrm{cu}]_{s=1}[\mathrm{bu}]_{s=0} \bar{c}$} & 10.21 & {$[\mathrm{cu}]_{s=1}[\mathrm{bu}]_{s=0} \bar{b}$} & 13.72 \\
{$[\mathrm{cu}]_{s=0}[\mathrm{bu}]_{s=1} \bar{c}$} & 10.22 & {$[\mathrm{cu}]_{s=0}[\mathrm{bu}]_{s=1} \bar{b}$} & 13.73 \\
{$[\mathrm{cs}]_{s=0}[\mathrm{bs}]_{s=1} \bar{c}$} & 10.64 & {$[\mathrm{cs}]_{s=0}[\mathrm{bs}]_{s=1} \bar{b}$} & 14.15 \\
{$[\mathrm{cs}]_{s=1}[\mathrm{bs}]_{s=0} \bar{c}$} & 10.65 & {$[\mathrm{cs}]_{s=1}[\mathrm{bs}]_{s=0} \bar{b}$} & 14.16 \\
{$[\mathrm{bu}]_{s=1}[\mathrm{bu}]_{s=0} \bar{c}$} & 13.33 & {$[\mathrm{bu}]_{s=1}[\mathrm{bu}]_{s=0} \bar{b}$} & 16.86 \\
{$[\mathrm{bs}]_{s=1}[\mathrm{bs}]_{s=0} \bar{c}$} & 13.67 & {$[\mathrm{bs}]_{s=1}[\mathrm{bs}]_{s=0} \bar{b}$} & 17.2 \\
\hline
\end{tabular}

TABLE VI. The masses of pentaquarks at $J^{p}=(3 / 2)^{-}$considering $\bar{q}=\bar{c}$ or $\bar{b}$ in $\mathrm{GeV}$.

\begin{tabular}{cccc}
\hline Pentaqurk & $\begin{array}{c}M \\
\text { this work) }\end{array}$ & Pentaquark & $\begin{array}{c}M \\
\text { (this work) }\end{array}$ \\
\hline$[\mathrm{cu}]_{s=1}[\mathrm{cu}]_{s=0} \bar{c}$ & 7.12 & {$[\mathrm{cu}]_{s=1}[\mathrm{cu}]_{s=0} \bar{b}$} & 10.61 \\
{$[\mathrm{cu}]_{s=0}[\mathrm{cs}]_{s=1} \bar{c}$} & 7.27 & {$[\mathrm{cu}]_{s=0}[\mathrm{cs}]_{s=1} \bar{b}$} & 10.76 \\
{$[\mathrm{cu}]_{s=1}[\mathrm{cs}]_{s=1} \bar{c}$} & 7.49 & {$[\mathrm{cu}]_{s=1}[\mathrm{cs}]_{s=1} \bar{b}$} & 10.99 \\
{$[\mathrm{cu}]_{s=1}[\mathrm{cs}]_{s=0} \bar{c}$} & 7.52 & {$[\mathrm{cu}]_{s=1}[\mathrm{cs}]_{s=0} \bar{b}$} & 11.02 \\
{$[\mathrm{cu}]_{s=1}[\mathrm{cu}]_{s=1} \bar{c}$} & 7.597 & {$[\mathrm{cu}]_{s=1}[\mathrm{cu}]_{s=1} \bar{b}$} & 11.09 \\
{$[\mathrm{cs}]_{s=1}[\mathrm{cs}]_{s=1} \bar{c}$} & 7.66 & {$[\mathrm{cs}]_{s=1}[\mathrm{cs}]_{s=1} \bar{b}$} & 11.15 \\
{$[\mathrm{cu}]_{s=1}[\mathrm{cs}]_{s=0} \bar{c}$} & 7.67 & {$[\mathrm{cu}]_{s=1}[\mathrm{cs}]_{s=0} \bar{b}$} & 11.18 \\
{$[\mathrm{cu}]_{s=1}[\mathrm{bu}]_{s=0} \bar{c}$} & 10.202 & {$[\mathrm{cu}]_{s=1}[\mathrm{bu}]_{s=0} \bar{b}$} & 13.71 \\
{$[\mathrm{cu}]_{s=0}[\mathrm{bu}]_{s=1} \bar{c}$} & 10.206 & {$[\mathrm{cu}]_{s=0}[\mathrm{bu}]_{s=1} \bar{b}$} & 13.72 \\
{$[\mathrm{cu}]_{s=1}[\mathrm{bu}]_{s=1} \bar{c}$} & 10.49 & {$[\mathrm{cu}]_{s=1}[\mathrm{bu}]_{s=1} \bar{b}$} & 14.01 \\
{$[\mathrm{cs}]_{s=1}[\mathrm{bs}]_{s=1} \bar{c}$} & 10.59 & {$[\mathrm{cs}]_{s=1}[\mathrm{bs}]_{s=1} \bar{b}$} & 14.11 \\
{$[\mathrm{cs}]_{s=0}[\mathrm{bs}]_{s=1} \bar{c}$} & 10.63 & {$[\mathrm{cs}]_{s=0}[\mathrm{bs}]_{s=1} \bar{b}$} & 14.14 \\
{$[\mathrm{cs}]_{s=1}[\mathrm{bs}]_{s=0} \bar{c}$} & 10.64 & {$[\mathrm{cs}]_{s=1}[\mathrm{bs}]_{s=0} \bar{b}$} & 14.15 \\
{$[\mathrm{bu}]_{s=1}[\mathrm{bu}]_{s=0} \bar{c}$} & 13.34 & {$[\mathrm{bu}]_{s=1}[\mathrm{bu}]_{s=0} \bar{b}$} & 16.86 \\
{$[\mathrm{bu}]_{s=1}[\mathrm{bu}]_{s=1} \bar{c}$} & 13.34 & {$[\mathrm{bu}]_{s=1}[\mathrm{bu}]_{s=1} \bar{b}$} & 16.86 \\
{$[\mathrm{bs}]_{s=1}[\mathrm{bs}]_{s=1} \bar{c}$} & 13.66 & {$[\mathrm{bs}]_{s=1}[\mathrm{bs}]_{s=1} \bar{b}$} & 17.18 \\
{$[\mathrm{bs}]_{s=1}[\mathrm{bs}]_{s=0} \bar{c}$} & 13.68 & {$[\mathrm{bs}]_{s=1}[\mathrm{bs}]_{s=0} \bar{b}$} & 17.2 \\
\hline
\end{tabular}


TABLE VII. The masses of pentaquarks at $J^{p}=(5 / 2)^{-}$considering $\bar{q}=\bar{c}$ or $\bar{b}$ in $\mathrm{GeV}$.

\begin{tabular}{cccc}
\hline Pentaqurk & $\begin{array}{c}M \\
\text { (this work) }\end{array}$ & Pentaquark & $\begin{array}{c}M \\
\text { (this work) }\end{array}$ \\
\hline$[\mathrm{bs}]_{s=1}[\mathrm{bs}]_{s=1} \bar{c}$ & 13.33 & {$[\mathrm{bs}]_{s=1}[\mathrm{bs}]_{s=1} \bar{b}$} & 16.85 \\
{$[\mathrm{bu}]_{s=1}[\mathrm{bu}]_{s=1} \bar{c}$} & 13.65 & {$[\mathrm{bu}]_{s=1}[\mathrm{bu}]_{s=1} \bar{b}$} & 17.17 \\
{$[\mathrm{bs}]_{s=1}[\mathrm{cs}]_{s=1} \bar{c}$} & 10.57 & {$[\mathrm{bs}]_{s=1}[\mathrm{cs}]_{s=1} \bar{b}$} & 14.08 \\
{$[\mathrm{bu}]_{s=1}[\mathrm{cu}]_{s=1} \bar{c}$} & 10.47 & {$[\mathrm{bu}]_{s=1}[\mathrm{cu}]_{s=1} \bar{b}$} & 13.98 \\
{$[\mathrm{cu}]_{s=1}[\mathrm{cu}]_{s=1} \bar{c}$} & 7.51 & {$[\mathrm{cu}]_{s=1}[\mathrm{cu}]_{s=1} \bar{b}$} & 11.02 \\
{$[\mathrm{cs}]_{s=1}[\mathrm{cs}]_{s=1} \bar{c}$} & 7.57 & {$[\mathrm{cs}]_{s=1}[\mathrm{cs}]_{s=1} \bar{b}$} & 11.08 \\
\hline
\end{tabular}

masses using the Bethe-Salpeter Eq. (3) for a diquark-diquark system with the considering potential, and then the mass of heavy pentaquarks at $\left((1 / 2)^{-},(3 / 2)^{-}\right.$, and $\left.(5 / 2)^{-}\right)$spin resulting from the four-quark state-to-one antiquark interaction. Where $m_{1}$ and $m_{2}$ are the masses of two diquarks and antiquark, and $M$ is the pentaquark mass. The masses of pentaquarks are shown in Tables II, III, and IV, with $l=0$ set and our results compared to Refs. [34,35]; notice that all states have negative parity. Other pentaquark masses with the same model but different antiquarks (charm quark or bottom quark) are mentioned in Tables V, VI, and VII.

Table II shows the pentaquark masses with the spin $(1 / 2)^{-}$and negative parity, based on the results of Table I. The same results are reported in Table III, but for pentaquarks with the spin $(3 / 2)^{-}$. Table IV also contains the results for the spin $(5 / 2)^{-}$pentaquarks. Pentaquarks with spin $(1 / 2)^{-}$ have masses ranging from 4.21 to $11.44 \mathrm{GeV}$, while those with spin $(3 / 2)^{-}$and $(5 / 2)^{-}$have masses ranging from 5.35 to 11.78 and 4.77 to $11.21 \mathrm{GeV}$, respectively.

\section{Conclusion}

We used the Bethe-Salpeter equation to describe pentaquark which contains two heavy-valence quarks within the present potential, which includes logarithm and linear potentials with a spin-spin interaction. As shown in Fig. 2, the method involves computing diquark masses from interactions between single quarks, followed by the four-quark state mass formed by two diquarks, and finally the pentaquark mass resulting from the four-quark state mass with an antiquark. Our pentaquark masses are mentioned in Tables II, III, IV, V, VI, and VII with spin $\left((1 / 2)^{-},(3 / 2)^{-}\right.$and $\left.(5 / 2)^{-}\right)$. In comparison to other recent works, the present potential produces compatible results with other potential models.
1. M. Abu-Shady, Chiral logarithmic quark model of $N$ and $\Delta$ with an A-term in the mean-field approximation, Int. J. Mod. Phys. A 26 (2011) 235, https://doi.org/10.1142/ S0217751X11051469

2. M. Abu-Shady, Effect of logarithmic mesonic potential on nucleon properties, Mod. Phys. Lett. A 24 (2009) 1617, https: //doi.org/10.1142/S0217732309030278

3. M. Abu-Shady, The effect of finite temperature on the nucleon properties in the extended linear sigma model, Int. J. Mod. Phys. E 21 (2012) 1250061, https: / / doi.org/10. 1142/S0218301312500619

4. M. Abu-Shady, Nucleon Properties Below the Critical Point Temperature, Int. J. Theor. Phys. 50 (2011) 1372, https: //doi.org/10.1007/s10773-010-0646-1.

5. M. Abu-Shady and E. M. Khokha, Heavy-Light Mesons in the Nonrelativistic Quark Model Using Laplace Transformation Method, Adv. High Energy Phys. 2018 (2018) 7032041, https://doi.org/10.1155/2018/7032041

6. M. Abu-Shady and Sh. Y. Ezz-Alarab, Trigonometric RosenMorse Potential as a Quark-Antiquark Interaction Potential for Meson Properties in the Nonrelativistic Quark Model Using EAIM, Few-Body Syst. 60 (2019) 66, https://doi.org/ $10.1007 / \mathrm{s} 00601-019-1531-\mathrm{y}$

7. M. Gell-Mann, A schematic model of baryons and mesons, Phys. Lett. 8 (1964) 214, https://doi.org/10.1016/ S0031-9163(64)92001-3.

8. R. L. Jaffe, Exotica, Phys. Rep. 409 (2005) 1, https: // doi. org/10.1016/j.physrep.2004.11.005, Nucl. Phys.
B Proc. Suppl. 142 (2005) 343, https://doi.org/10. $1016 / j . n u c l p h y s b p s .2005 .01 .058$

9. M. Yu. Barabanov et al., Diquark correlations in hadron physics: Origin, impact and evidence, Prog. Part. Nucl. Phys. 116 (2021) 103835, https: //doi.org/10.1016/ j.ppnp.2020.103835

10. R. J. Jaffe, Multiquark hadrons I. Phenomenology of $Q^{2} \bar{Q}^{2}$ mesons, Phys. Rev. D 15 (1977) 267, https://doi.org/ $10.1103 /$ PhysRevD.15.267

11. M. Tanabashi et al., (Particle Data Group), Review of Particle Physics, Phys. Rev. D 98 (2018) 030001, https: / / doi. org/10.1103/PhysRevD.98.030001

12. P. Colangelo, F. De Fazio, F. Giannuzzi, and S. Nicotri, New meson spectroscopy with open charm and beauty, Phys. Rev. D 86 (2012) 054024, https://doi.org/10.1103/ PhysRevD.86.054024

13. A. Ali, J. S. Lange, and S. Stone, Exotics: Heavy pentaquarks and tetraquarks, Prog. Part. Nucl. Phys. 97 (2017) 123, https://doi.org/10.1016/j.ppnp.2017.08. 003

14. M. Karliner, J. L. Rosner, and T. Skwarnicki, Multiquark States, Annu. Rev. Nucl. Part. Sci. 68 (2018) 17, https://doi. org/10.1146/annurev-nucl-101917-020902

15. H.-X. Chen, W. Chen, X. Liu, and S.-L. Zhu, The hiddencharm pentaquark and tetraquark states, Phys. Rep. 639 (2016) 1, https://doi.org/10.1016/j.physrep. 2016.05 .004 
16. S.-K. Choi et al., (Belle Collaboration), Observation of a Narrow Charmoniumlike State in Exclusive $B^{ \pm} \rightarrow K^{ \pm} \pi^{+} \pi^{-} J / \psi$ Decays, Phys. Rev. Lett. 91 (2003) 262001, https://doi. org/10.1103/PhysRevLett.91.262001

17. S. J. Brodsky and R. F. Lebed, QCD dynamics of tetraquark production, Phys. Rev. D 91 (2015) 114025, https: / / doi. org/10.1103/PhysRevD.91.114025

18. R. Aaij et al. (LHCb Collaboration), Observation of $J / \psi p$ Resonances Consistent with Pentaquark States in $\Lambda_{b}^{0} \rightarrow J / \psi K^{-} p$ Decays, Phys. Rev. Lett. 115 (2015) 072001, https : / / doi. org/10.1103/PhysRevLett.115.072001.

19. R. Zhu, Fully-heavy tetraquark spectra and production at hadron colliders, Nucl. Phys. B 966 (2021) 115393, https: //doi.org/10.1016/j.nuclphysb.2021.115393

20. C. Becchi, J. Ferretti, A. Giachino, L. Maiani, and E. Santopinto, A study of $c c \overline{c c}$ tetraquark decays in 4 muons and in $D^{(*)} \bar{D}^{(*)}$ at LHC, Phys. Lett. B 811 (2020) 135952, https : //doi.org/10.1016/j.physletb.2020.135952

21. S. L. Olsen, T. Skwarnicki, and D. Zieminska, Nonstandard heavy mesons and baryons: Experimental evidence, Rev. Mod. Phys. 90 (2018) 015003, https://doi.org/10.1103/ RevModPhys.90.015003

22. R. Zhu and C.-F. Qiao, Pentaquark states in a diquark-triquark model, Phys. Lett. B 756 (2016) 259, https://doi.org/ $10.1016 / j \cdot$ physletb.2016.03.022

23. Z.-G. Wang, Analysis of the doubly heavy baryon states and pentaquark states with QCD sum rules, Eur. Phys. J. C 78 (2018) 826, https://doi.org/10.1140/epjc/ s10052-018-6300-4.

24. Q.-S. Zhou, K. Chen, X. Liu, Y.-R. Liu, and S.-L. Zhu, Surveying exotic pentaquarks with the typical $Q Q q q \bar{q}$ configuration, Phys. Rev. C 98 (2018) 045204, https://doi.org/10. 1103/PhysRevC.98.045204

25. R. Aaij et al., (LHCb Collaboration), Observation of a Narrow Pentaquark State $P_{c}(4312)^{+}$, and of the Two-Peak Structure of the $P_{c}(4450)^{+}$, Phys. Rev. Lett. 122 (2019) 222001, https : //doi.org/10.1103/PhysRevLett.122.222001

26. R. Aaij et al. (LHCb Collaboration), Model-Independent Evidence for $J / \psi p$ Contributions to $\Lambda_{b}^{0} \rightarrow J / \psi p K^{-}$Decays, Phys. Rev. Lett. 117 (2016) 082002, https://doi.org/ 10.1103/PhysRevLett.117.082002

27. T. J. Burns and E. S. Swanson, Molecular interpretation of the $P_{c}(4440)$ and $P_{c}(4457)$ states, Phys. Rev.
D 100 (2019) 114033, https://doi.org/10.1103/ PhysRevD.100.114033.

28. R. D. Matheus, F. S. Navarra, M. Nielsen, and R. Rodrigues da Silva, Pentaquark masses in QCD sum rules, Nucl. Phys. B Proc. Suppl. 152 (2006) 228, https://doi.org/10. $1016 / j$.nuclphysbps.2005.08.043

29. T. Inoue, V. E. Lyubovitskij, Th. Gutsche, and A. Faessler, Mass spectrum of the $J^{P}=1 / 2^{-}$and $3 / 2^{-}$pentaquark antidecuplets in the perturbative chiral quark model, Int. J. Mod. Phys. E 14 (2005) 995, https://doi.org/10.1142/ S0218301305003752

30. E. Santopinto and A. Giachino, Compact pentaquark structures, Phys. Rev. D 96 (2017) 014014, https://doi.org/10. 1103/PhysRevD.96.014014

31. R. Bijker, M. M. Giannini, and E. Santopinto, Spectroscopy of pentaquark states, Eur. Phys. J. A 22 (2004) 319, https: //doi.org/10.1140/epja/i2003-10232-x

32. M. Karliner and H. J. Lipkin, A diquark-triquark model for the $K N$ pentaquark, Phys. Lett. B 575 (2003) 249, https : //doi.org/10.1016/j.physletb.2003.09.062

33. L. Maiani, A. D. Polosa, and V. Riquer, The new pentaquarks in the diquark model, Phys. Lett. B 749 (2015) 289, https : //doi.org/10.1016/j.physletb.2015.08.008

34. F. Giannuzzi, Heavy pentaquark spectroscopy in the diquark model, Phys. Rev. D 99 (2019) 094006, https://doi. org/10.1103/PhysRevD.99.094006

35. S. M. M. Nejad and A. Armat, Determination of the Mass and the Energy Spectra of Heavy Pentaquarks in the Diquark Model, Few-Body Syst. 61 (2020) 31, https://doi.org/ 10.1007/s00601-020-01564-2

36. Y. Chargui, On an approximation of the two-body spinless Salpeter equation, Eur. Phys. J. Plus 133 (2018) 543, https : //doi.org/10.1140/epjp/i2018-12420-4.

37. S. Rahmani, H. Hassanabadi, and H. Sobhani, Mass and decay properties of double heavy baryons with a phenomenological potential model, Eur. Phys. J. C 80 (2020) 312, https: //doi.org/10.1140/epjc/s10052-020-7867-0

38. A. Armat and H. Hassanabadi, Study of ground state binding energies of the single $\Xi$ and $\Lambda$ hypernuclei by using numerical computation, Can. J. Phys. 95 (2017) 1086, https: //doi.org/10.1139/cjp-2017-0011 